ror

SECCIÓN: Teorías geográficas, geografía de la cultura y la vida cotidiana
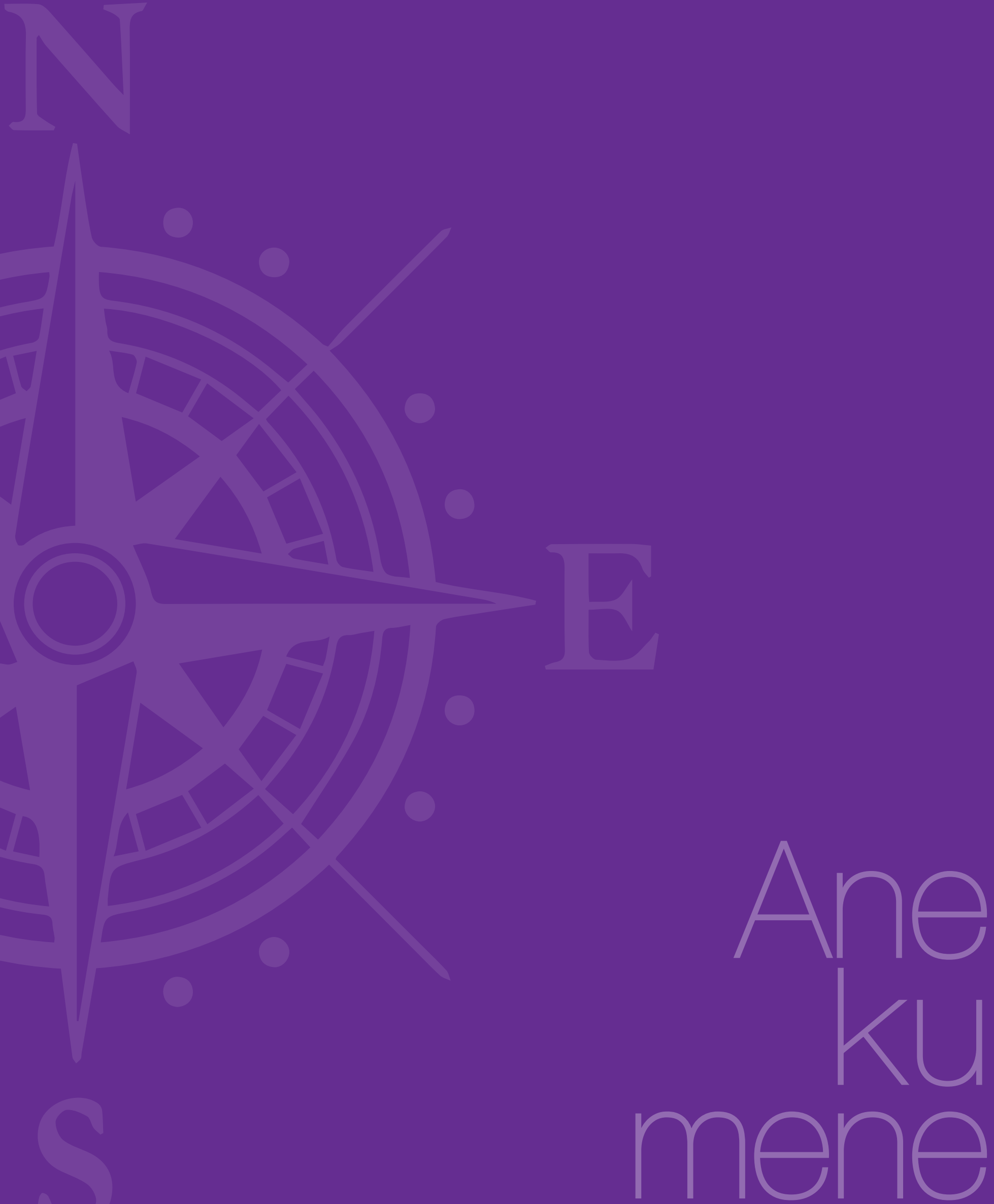


\section{O papel das geotecnologias no desenvolvimento do pensamento espacial}

El papel de las geotecnologías en el desarrollo del pensamiento espacial

\section{The Role of Geotechnologies in the Development of Spatial Thinking}

Danúbia Caporusso Bargos*

Lindon Fonseca Matias**

\section{Resumo}

O pensamento espacial pode ser considerado uma distinta forma de pensar que congrega conceitos de espaço, instrumentos de representação e processos de raciocínio, sendo seu desenvolvimento considerado fundamental para a formação de cidadãos críticos e capazes de compreender e transformar a realidade em que vivem. As geotecnologias por sua vez são consideradas importantes instrumentos para análise e compreensão do espaço geográfico e para o desenvolvimento do pensamento espacial. 0 texto aqui apresentado, em contribuição da coordenação da mesa-redonda "A Cartografia Temática, o Raciocínio Geográfico e a Cidade”, busca refletir sobre o desenvolvimento desse tipo de pensamento e sua expressão por meio do uso das geotecnologias na contemporaneidade. Partindo de diferentes definições e concepções ligadas ao pensamento espacial e de um debate sobre o reconhecimento de uma Ciência da Informação Geográfica, é apresentada uma discussão destinada a demonstrar as motivações, influência e os desdobramentos da aplicação das geotecnologias no desenvolvimento do pensamento espacial crítico. Considera-se que, apesar dos avanços tecnológicos e do crescente uso das geotecnologias, tanto no ambiente escolar como instrumentos auxiliares das práticas didático-pedagógicas, ainda é necessário um aprofundamento na base epistemológica das discussões ligadas às geotecnologias, considerando que seu uso não resume um simples tratamento instrumental, mas como uma nova maneira de fazer e pensar a geografia.

\section{Palavras-chave}

Pensamento espacial, geotecnologias, Ciência da Informação Espacial.

\footnotetext{
* Escola de Engenharia de Lorena-usp.

** Universidade Estadual de Campinas.
} 


\section{Resumen}

El pensamiento espacial puede considerarse una forma distinta de pensar que congrega conceptos de espacio, instrumentos de representación y procesos de raciocinio. Su desarrollo es fundamental para la formación de ciudadanos críticos capaces de comprendery transformar la realidad en que viven. Las geotecnologías, a su vez, se consideran importantes instrumentos para el análisis y la comprensión del espacio geográfico y para el desarrollo del pensamiento espacial. El texto aquí presentado, como contribución a la coordinación de la mesa redonda "La Cartografía Temática, el Razonamiento Geográfico y la Ciudad”, busca reflexionar frente al desarrollo de este tipo de pensamiento y su expresión por medio del uso de las geotecnologías en la contemporaneidad. A partir de diferentes definiciones y concepciones ligadas al pensamiento espacial y de un debate en torno al reconocimiento de una ciencia de la información geográfica, se presenta una discusión orientada a evidenciar las motivaciones, la influencia y el despliegue de la aplicación de las geotecnologías en el desarrollo del pensamiento espacial crítico. Se considera que, a pesar de los avances tecnológicos y del creciente uso de las geotecnologías, tanto en el ambiente escolar como en los instrumentos auxiliares de las prácticas pedagógicas, todavía se requiere de una profundización en las discusiones de base epistemológica vinculadas a las geotecnologías para que su uso trascienda el ámbito instrumental y se consideren, por el contrario, como una nueva forma de hacer y pensar geografía.

\section{Abstract}

Spatial thinking can be considered a different way of thinking that brings together the concepts of space, representation tools, and processes of reasoning. It is essential to form critical citizens who are able to understand and transform their reality. Geotechnologies, in turn, are important tools to anzlyze and understand the geographical space and to develop spatial thinking. This text was a contribution to the Thematic Cartography, Geographical Reasoning and the City Roundtable. Its purpose is to reflect on the development of this type of thinking and its expression through the use of geotechnologies in the contemporary world. Based on different definitions and concepts linked to spatial thinking and a debate about the recognition of a Geographic Information Science, the authors show the motivations, influence and spread out of the application of geotechnologies in the development of critical spatial thinking. The researchers conclude that, in spite of the technological advances and the increasing use of geotechnologies-both in the school environment and in the auxiliary instruments of pedagogical practices-, a deeper epistemological debate on this issue is still required. This would allow their use to go beyond instrumentalization, and approach them as a new way of doing and thinking geography.

\section{Palabras clave}

Pensamiento espacial, geotecnologías, ciencia de la información espacial.

\section{Keywords}

Spatial thinking, geotechnologies, Spatial Information Science. 


\section{Introdução}

Discutir o papel das geotecnologias no desenvolvimento do pensamento espacial não é uma tarefa simples; pois, embora envolva temas já bastante discutidos, principalmente no âmbito da Geografia, pouco se discute sobre a efetividade dessa relação. Normalmente o debate está centrado na utilização de um instrumental ou aparato tecnológico, quase sempre o uso de um determinado software e hardware, para a abordagem de uma problemática específica que envolve questões de localização, orientação, escala, dentre outros; mas não envolve a mediação da técnica na construção do pensamento espacial. Assim, buscando outra orientação para essa discussão, o ponto de partida aqui proposto é a definição de termos que sustentam a compreensão da totalidade do pensamento espacial.

Para Maciel e Pinheiro (2013), o termo inteligência é empregado em diversas disciplinas, sendo que no contexto da Ciência da Informação esta pode ser considerada como uma atividade sistemática de coleta e análise de informações necessárias à produção do conhecimento e ação, enquanto na Psicologia considera-se as habilidades cognitivas de um individuo. 0 termo habilidade, rotineiramente recorrente em textos pedagógicos e em materiais de orientação de ensino, refere-se por sua vez às competências adquiridas pela prática para realização de tarefas ou às aptidões do ser humano para resolução de problemas e tomada de decisões diante de distintas situações; e, implica no desenvolvimento de capacidades para realização de tarefas de forma mais rápida ou ágil do que aquelas realizadas na ausência do exercício dos procedimentos que conduzem o raciocínio das ações (Ferreira, 2013).

No caso da Geografia, como em outras ciências, o processo de aprendizagem implica no desenvolvimento de uma infinidade de aptidões, tais como: fazer relações entre fatos e fenômenos; interpretar imagens, textos, símbolos e representações; formular ideias a partir de saberes sobre como a sociedade e a natureza se organizam no plano espacial (Ferreira, 2013, p. 72).

O desenvolvimento das aptidões apontadas por Ferreira (2013) relaciona-se diretamente com o desenvolvimento do raciocínio que, dentre outros, confere sentido ao pensamento e auxilia na construção do conhecimento. Vale lembrar que, para Jean Piaget (1983) o conhecimento evolui progressivamente sendo resultante da interação/experiência do sujeito com o objeto. No âmbito da Ciência da Informação o conhecimento pode ser hierarquizado a partir de um modelo teórico denominado DIKW (Data-Information-Knowledge-Wisdom) ou Pirâmide do Conhecimento.

A Pirâmide do conhecimento é composta por extratos que permitem maior compreensão à medida que se elevam os níveis de abstração e expressa resumidamente a estrutura necessária para a compreensão ou interpretação do mundo. Os dados, que compõem a base desta estrutura e, portanto, o seu nível mais básico, são códigos que isoladamente e/ou não processados são incapazes de produzir qualquer conhecimento. A informação confere significado ao dado e é pressuposto do conhecimento que, diferentemente da informação, exige trabalho intelectual de formulação e teste de hipóteses. Por fim, a sabedoria é alcançada quando se atinge o mais alto nível da relação entre abstração e compreensão que se constrói a partir da experiência, principalmente como base da resolução de problemas existentes na realidade com elevado grau de acertos.

Apesar de a hierarquia DIKw não ser a única, tão pouco a mais antiga ou aceita teoria para compreensão da construção do conhecimento, considera-se que ela pode ser utilizada para problematização da discussão que envolve o emprego das geotecnologias no desenvolvimento do pensamento espacial partindo de questões que buscam responder como a técnica poderia atuar como mediadora desse processo. Antes disso, porém, faz-necessário definir o que se entende por pensamento espacial.

As distintas formas de pensamento (verbal, lógico, metafórico, hipotético, matemático etc.), podem ser diferenciadas em termos de seu sistema representacional ou de raciocínio. Assim, o pensamento verbal faz uso de símbolos linguísticos e o matemático de símbolos matemáticos, por exemplo; sendo que em qualquer domínio do conhecimento são usadas múltiplas formas de pensamento. O pensamento espacial é uma forma distintiva de pensar, sendo o conceito de espaço o que faz do pensamento espacial uma forma diferente de pensamento (NRC, 2006), que se constrói a partir de uma coleção de habilidades cognitivas que consistem em formas declarativas e perceptivas de conhecimento e algumas operações cognitivas que podem ser utilizadas para transformar, combinar ou operar sobre esse conhecimento (Zwartjes, Lázaro, Donert, Sanchez, Gonzalez e Wiśniewska, 2016).

Conforme Duarte (2017), o núcleo da definição do conceito de pensamento espacial que vem sendo reconhecido pela comunidade acadêmica voltada ao estudo do pensamento espacial é encontrado no relatório "Learning to Think Spatially: GIS as a Support System in the K-12 Curriculum" elaborado pelo Committee on the Support for the Thinking Spatially ligado à Comissão de Geografia do National Research Council (NRC) dos Estados Unidos. Neste documento o pensamento espacial, enquanto forma de pensamento, é uma forma distintiva de pensar baseada na junção de três elementos: (1) conceitos de espaço, (2) instrumentos de representação e (3) processos de raciocínio (NRC, 2006). Assim, pensar espacialmente implica saber, por exemplo, sobre (1) relações entre unidades de medida, diferentes formas de calcular a distância, base de sistemas de coordenadas, natureza dos espaços; (2) relações entre visualizações, plano versus elevação, mapas ortogonais versus mapas de perspectiva, efeito das projeções, princípios de design gráfico; (3) diferentes maneiras de pensar sobre as distâncias mais curtas, capacidade de extrapolar e interpolar e tomar decisões, dentre outros (NRC, 2006, p. 12). 
Apesar do reconhecimento desta definição e em função de todo o desenvolvimento prático e científico que envolve o termo pensamento espacial, uma variedade de termos a ele relacionados, tais como alfabetização, habilidade e raciocínio espacial, surgiu nos últimos anos e contribuem para o aumento da imprecisão da definição deste termo (Wakabayashi e Ishikawa, 2011). Buscando facilitar a compreensão da diferenciação e dependência entre estes termos, Wakabayashi e Ishikawa (2011) propõem um esquema conceitual dos termos relacionados ao pensamento espacial onde as habilidades espaciais compõem a base de uma pirâmide que sustenta na sequência o pensamento espacial-composto por conceitos, representações e raciocínio; e, ao final, a alfabetização espacial.

Assim como na hierarquia DIKW, o topo da pirâmide, neste caso a alfabetização ou inteligência espacial, somente é alcançado quando se atinge o mais alto nível da relação entre abstração e compreensão que se dá a partir da experiência (Figura 1). Numa perspectiva parecida, Tuan (1983) defende que a habilidade espacial se transforma em conhecimento espacial quando podem ser intuídos os movimentos e as mudanças de localização.

Figura 1. Pirâmide da gestão do conhecimento e informação.

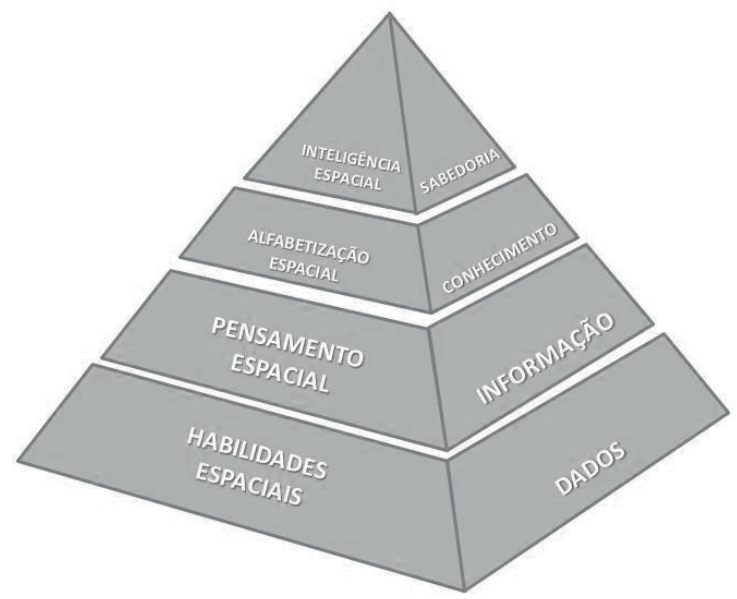

Fonte: Organizado pelos autores. Adaptado de Wakabayashi e Ishikawa (2011).

O raciocínio espacial por sua vez, está envolvido no processo cognitivo de ordem superior da inferência de conhecimento prévio para resolver problemas ou tomar decisões, podendo então ser classificado em três níveis: entrada (com coleta de informação dos sentidos ou informações de memórias); processamento (análise, classificação, explicação ou comparação de informações adquiridas no nível anterior); e saída (geração de novos conhecimentos ou produtos a partir das informações obtidas nos dois primeiros níveis) (Wakabayashi e Ishikawa, 2011).

\section{Pensamento Espacial: Conceitos, Definições e Discussões}

O pensamento espacial é uma das formas fundamentais de inteligência humana necessárias para a sociedade moderna cujo desenvolvimento deve fazer parte da educação de todos, assim como linguística ou matemática; e deve ser ensinado e aprendido na escola e na vida cotidiana (Goodchild, 2006). Na escola, não apenas na Geografia, que desempenha um importante papel de aplicação do pensamento espacial, mas também nas outras disciplinas, como nas ciências e matemática. Na vida cotidiana, usando o espaço como uma metáfora ou analogia e colocando informações não espaciais em um contexto espacial para exibir, resumir e estimular a análise e o pensamento com o espaço; por meio, por exemplo, de mapas conceituais, gráficos climáticos, pirâmides populacionais. Neste contexto, vale lembrar que uma das características distintivas dos seres humanos é que eles podem usar representações simbólicas, como linguagem, mapas, diagramas, esboços e gráficos (Newcombe, 2010).

A linguagem espacial pode ser considerada um importante instrumento para a aprendizagem espacial. Conforme Newcombe (2010) o pensamento espacial das crianças é influenciado pelo ambiente doméstico e pode melhorar com brincadeiras e jogos de computador; bebês aprendem melhor uma relação espacial quando recebem um nome; pré-escolares que entendem palavras espaciais como "meio" têm melhor desempenho em tarefas espaciais do que aquelas que não usam, e crianças pré-escolares cujos pais usam um maior número de palavras espaciais (sob, sobre, ao redor, canto, dentre outras) mostram melhor desenvolvimento do pensamento espacial do que crianças cujos pais não usam essa linguagem.

Para Boroditsky (2018) a linguagem modela a forma como os humanos pensam e pode inclusive influenciar no desenvolvimento da orientação. Como exemplo, a autora cita o caso de uma comunidade aborígene da Austrália (Kuuk Thaayorre) onde as pessoas não usam palavras como "esquerda" e "direita" para referência a direções e localizações, mas ao invés disso, expressam-se com pontos cardeais. Assim, com base nas observações dos hábitos, costumes e rotinas desse povo, a pesquisadora considera que as pessoas dessa comunidade são capazes de desenvolver o senso de orientação mais rapidamente que povos que se utilizam de outras formas de linguagem.

Newcombe (2010) considera que o pensamento espacial dos adultos também é aprimorado pela linguagem espacial assim como o pensamento deles sobre conceitos como o tempo, que são frequentemente descritos com metáforas espaciais. Na mesma perspectiva, a capacidade de usar mapas pode transformar o pensamento, permitindo conclusões que seriam difíceis de chegar sem eles. Um exemplo famoso é ver a relação entre beber água poluída e contrair cólera; em 1800, um mapa de bombas de água em Londres sobreposto a um mapa de casos de cólera poderia revelar essa relação (Newcombe, 2010). 
De forma geral, considerando o que fora até aqui exposto, torna-se imprescindível destacar o papel da linguagem cartográfica no desenvolvimento do pensamento e raciocínio espacial; pois esta, conforme Castellar (2017) “(...) estrutura-se em símbolos e signos, sendo compreendida como um produto da comunicação visual que dissemina informação espacial” (p. 221).

Kerski (2008) resume o pensamento espacial como a capacidade de estudar as características e os processos interconectados da natureza e do impacto humano no tempo e em escalas apropriadas. Para Bednarz e Lee (2011) o pensamento espacial não é uma habilidade única, mas compõe-se de uma coleção de habilidades diferentes, em que componentes de pensamento espacial emergem: visualização e sobreposição de mapas, identificação e classificação de símbolos de mapa (ponto, linha, área), uso de operações booleanas, navegação de mapas e reconhecimento de correlação espacial. Já para Castellar (2017) o pensamento espacial é “(...) complexo, requer observar, comparar, relacionar, analisar, argumentar e elaborar crítica" (p. 210), enquanto para Duarte (2017) essa dimensão da inteligência é fundamental para a cidadania e para a prática de muitas atividades profissionais e cotidianas.

Na dimensão do ensino, o pensamento espacial sempre foi uma habilidade cognitiva fundamental para a aprendizagem na Geografia (Metoyer, Bednarz S. e Bednarz R., 2015) e desempenha três funções específicas: descritiva, voltada para a captura, a preservação e a transmissão das aparências e relações entre objetos; analítica, que permite compreender a estrutura de objetos; e, inferencial que auxilia na dedução de respostas a questões sobre a evolução e função de objetos (NRC, 2006). Em outras palavras, ou resumidamente, o pensamento espacial está diretamente relacionado à capacidade de apropriação, compreensão e aplicação das principais categorias (forma, função, estrutura e processo) eleitas por Santos (1978) para análise do espaço geográfico.

Dessa forma, o pensamento espacial não é estático, tratando-se de um processo dinâmico que permite descrever, explicar e prever a estrutura e funções dos objetos e suas relações em mundos reais ou imaginários; o que permite a elaboração de hipóteses e previsões (NRC, 2006). Deve ser crítico, considerando os processos mentais que acompanham o uso de procedimentos teóricos e metodológicos diversos e de dados espaciais, e promover, não apenas o pressionar de botões na interface de um aplicativo, mas o pensamento crítico sobre questões como escala, precisão, incerteza, ontologia, representação, complexidade, projeção e ética (Goodchild e Janelle, 2010). Também não se restringe a nenhum domínio de conhecimento ou se apresenta superior ou inferior a qualquer tipo de pensamento; mas é, conforme Duarte (2016), indiscutivelmente carregado de singularidades sendo uma forma de pensar que "(...) pode ser a mais adequada para compreender muitos fenômenos naturais e sociais, notadamente aqueles em que a espacialidade possui papel explicativo relevante" (Duarte, 2016, p. 86). Ainda para o mesmo autor, o processo de desenvolvimento do pensamento espacial tem como fatores fundamentais o domínio de habilidades intelectuais relacionadas à capacidade de operar com conceitos espaciais e com representações espaciais internas e externas.

De acordo com os pesquisadores do National Research Council o pensamento espacial pode ser base para uma expertise sofisticada ou para julgamentos cotidianos. Para eles todos podem e pensam espacialmente, apesar de o fazerem de maneiras diferentes. Algumas pessoas são mais bem sucedidas no pensamento espacial apenas em um domínio limitado de conhecimento enquanto outras podem ser capazes de lidar com uma ampla gama de problemas espaciais (NRC, 2006).

Nesta perspectiva, é importante ressaltar que o desenvolvimento da alfabetização espacial é importante e deve basear-se em habilidades práticas de ensino que são muitas vezes complexas e que existem como parte do envolvimento do educando em um processo de aprendizagem que incluem uma sequência de competências espaciais, tais como: orientação espacial (o componente "onde?"); pensar e agir (onde é em relação a?); o processo espacial (ou que mudanças estão ocorrendo?); os sistemas espaciais (ou como estão sendo afetados?); as questões mais amplas (ou como se conectam além de?); tomada de decisões (ou quais soluções existem?); e, como posso fazer a diferença? (Wakabayashi e Ishikawa, 2011).

Em referência ao relatório do NRC (2006), Wakabayashi e Ishikawa (2011) pontuam que educandos alfabetizados/inteligentes espacialmente desenvolvem níveis apropriados de conhecimento espacial e habilidades como formas de pensar e agir juntamente com um conjunto de capacidades espaciais. Assim, possuem o hábito mental de pensar espacialmente (sabem onde, quando, como e por que pensar espacialmente) e um amplo e profundo conhecimento de conceitos e representações espaciais que possibilita o comando sobre o raciocínio espacial usando uma variedade de modos espaciais de agir e pensar; o que lhes permite adotar uma postura crítica em relação pensamento espacial com capacidade para o engajamento, participação, ação e o exercício da cidadania espacial (Gryl, Jekel, e Donert, 2010). Donert (2008) sugere que o desenvolvimento das competências/habilidades espaciais deve conduzir a uma pedagogia ativa de aprendizagem onde a exploração (dos lugares) acontece, as medidas (associadas à localização) são coletadas e observações (de lugares) são feitas.

Goodchild e Janelle (2010) consideram que o desenvolvimento do pensamento espacial crítico está relacionado ao uso de instrumentos e dados espaciais e aos processos mentais que acompanham o uso dessas tecnologias. Para os autores, o pensamento espacial crítico está em nítido contraste com o ato mecânico de apertar botões, ou simples escolha de opções na tela, e implica que os processos de manuseio de dados, análise, mineração de dados e modelagem devem provocar e exigir pensamento crítico sobre questões comparativamente profundas como escala, 
precisão, incerteza, ontologia, representação, complexidade, projeção e ética. Desse modo, as tecnologias espaciais se apresentam como um elemento integrante essencial que transita entre diferentes disciplinas por meio de linguagem e conceitos comuns, sendo que um dos conceitos espaciais mais problemáticos é a escala, em ambos os seus significados duais de extensão e resolução (Goodchild e Janelle, 2010).

Para Gryl et al. (2010) um cidadão espacial ou uma pessoa alfabetizada espacialmente deve ser capaz de interpretar e refletir criticamente sobre representações espaciais, além de comunicar-se com o auxílio de mapas e outras representações espaciais. Os autores defendem que as comunicações espaciais podem ser consideradas parte integrante da cidadania espacial e, portanto, um alvo principal da educação primária e secundária, com abordagens diferentes para aquisição de competências técnicas necessárias aos alunos como cidadãos e de competências necessárias para a participação ativa e crítica. Além disso, sugerem ainda com base em Strobl (2008) uma diferenciação entre as necessidades de educação tecnológica para vários grupos-alvo de acordo com sua prática cotidiana. A partir daí, seria possível definir três tipos de sujeitos espacialmente capacitados/ alfabetizados ou com o pensamento espacial bem desenvolvido conforme os diferentes níveis educacionais: o cidadão espacial, o analista espacial e o gestor de sistemas de informações espaciais (Figura 2).

Figura 2. Esferas de atividades na geoinformação.

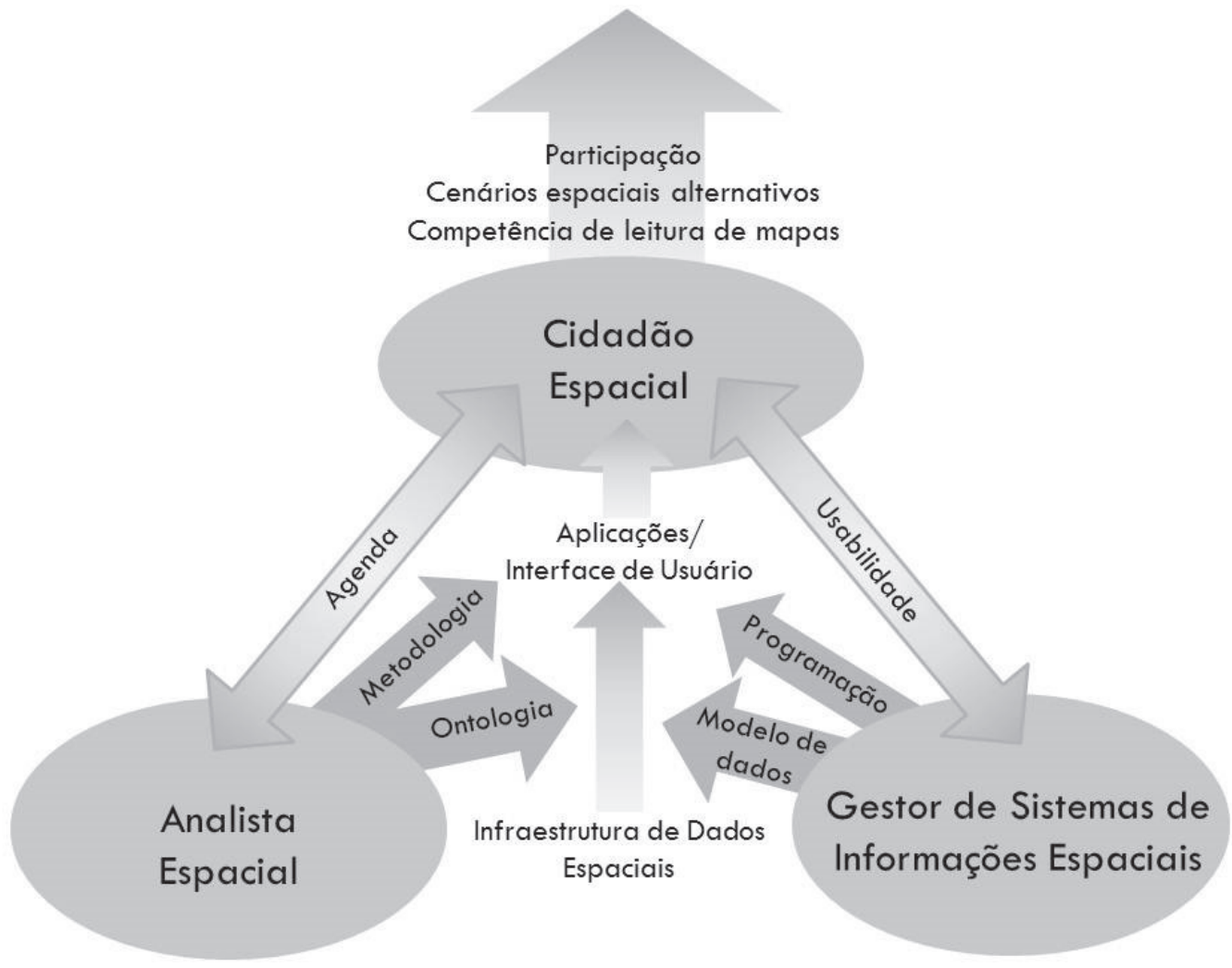

Fonte: Organizado pelos autores. Adaptado de Gryl et al. (2010). 


\section{Pensamento espacial, Geotecnologias e (Ciência da?) informação Geográfica}

A capacidade de abstração do espaço, a partir de conceitos geométricos (distância, coordenadas e dimensões) é fundamental para o desenvolvimento do pensamento espacial, cujas habilidades podem ser aprendidas dentro de um contexto específico com o apoio de diferentes ferramentas e tecnologias (NCR, 2006; Wakabayashi e Ishikawa, 2011). Para autores como Zwartjes et al. (2016), os indivíduos em processo de aprendizagem precisam conhecer os blocos de construção do pensamento espacial; o que inclui modelos, gráficos, fotografias, modelagem 3D, vídeo e outras ferramentas multimídia que podem ser apresentadas dentro de contextos específicos de aprendizagem e ser apoiados pelo uso de tecnologias geoespaciais, também chamadas de geotecnologias.

As geotecnologias podem ser consideradas uma categoria especial de tecnologia voltada para aquisição, processamento e análise e/ou disponibilização de dados geoespaciais e inclui a Cartografia Digital, o Sensoriamento Remoto, o Sistema de Posicionamento Global - GPS e o Sistema de Informações Geográficas - SIG (Esquerdo, Cruz, Macário, Antunes, Silva e Coutinho, 2014; Matias, 2004). Para Goodchild (2009) essas tecnologias levantam questões de importância fundamental formando um domínio da ciência cujas descobertas fornecem a base para as tecnologias: a GIScience (Ciência da Informação Geográfica).

O termo GIscience vem sendo usado por Michael F. Goodchild desde o início da década de 1990, sendo que ao longo dos anos outros termos como ciência geoespacial, geoinformática, geomática e ciência espacial foram surgindo, ora como sinônimos, ora como complementares ao termo GIscience. Para o autor, a ciência da informação geográfica pode ser definida como o estudo das questões fundamentais da informação geográfica e é frequentemente motivada pela necessidade de melhorar as tecnologias de informação geográfica (Goodchild, 2011).

Recentemente diversos termos como geodireito, geomarketing, geovisualização, geodesign, dentre outros foram criados e se associam ao corpo científico que possibilita o uso da informação geográfica, como a Geodésia, a Estatística, a Cartografia, além das ciências que receberam maior atenção a partir do século xx, como "(...) a ciência cognitiva para compreensão de como percebemos e representamos o espaço geográfico, a da informação para estudar o fenômeno informação, ou mesmo a ciência da computação no desenvolvimento de úteis tecnologias digitais" (Ramos, 2015, s. p).

Apesar da difusão do termo mais comumente utilizado, alguns autores consideram que a definição do que vem a ser a Ciência da Informação Geográfica é complexa e que das várias definições dadas ao longo dos anos poucas são totalmente satisfatórias (Blaschke e Merschdorf, 2014). Mesmo assim, para seu precursor muito progresso já foi feito no campo da GIScience, sendo esta “(...) amplamente reconhecida como um campo de pesquisa e um subconjunto bem definido da ciência da informação" (Goodchild, 2009, p.1037). Neste sentido, pode-se discutir um certo afastamento da ciência geográfica propriamente dita como faz, entre outros, Matias (2004) ao apontar a necessidade de avaliar até que ponto a GIScience desprende-se do campo da Geografia.

Blaschke e Merschdorf (2014) distinguem três perspectivas principais dos motivos pelos quais alguns pesquisadores consideram a GIscience como um campo das ciências. Para os autores, muitas das tentativas iniciais de definição da GIscience partiram de um déficit teórico e da ausência da demarcação de um campo específico da ciência relacionado às tecnologias geoespaciais, além da necessidade de uma abordagem multiparadigmática para essas tecnologias.

Goodchild (2004) tenta justificar a GIscience como ciência com base na sua dependência de leis e princípios científicos, concluindo que os méritos da GIscience como ciência são de fato atribuídos à base de tais e muitos outros princípios científicos. No entanto, ele admite que ainda não há consenso entre os pesquisadores quanto aos princípios subjacentes da GIscience. Embora o precursor desta ideia esteja convencido da validade da GIscience como um campo da ciência, baseado em sua fundamentação em princípios científicos, estes não são universalmente reconhecidos como critérios adequados (Blaschke e Merschdorf, 2014).

Ao discutir sobre a existência/definição da ciência da informação geográfica, Ramos (2015) argumenta que, se aceito o termo ciência da informação geográfica, seria esta uma ciência de caráter multidisciplinar que não define limite para seus domínios, mas promove a interação entre as abordagens de diferentes disciplinas. Em complemento ao argumento do autor, ressalta-se ainda que essa interação não se limita apenas a diferentes disciplinas, mas também às diferentes áreas do conhecimento.

Blaschke e Merschdorf (2014) argumentam que os laços estreitos da GIScience com as humanidades devem ser reconhecidos, tanto com base em sua natureza indutiva quanto com base em suas ligações temáticas com disciplinas das ciências sociais. Para Reitsma (2013) a GIscience não estuda o mundo como tal, em contraste com a maioria das outras ciências, mas sim as representações do mundo em termos de informações geográficas considerando os procedimentos envolvidos na coleta, gerenciamento, análise e visualização de tais informações. Assim, a GIScience não pode existir independentemente de outras ciências, mas sim em simbiose com outras disciplinas, como geografia e psicologia, que orientam a coleta de dados e outras necessidades de informação, sendo de natureza dicotômica, ao invés de ser um todo coerente, que pode ser colocado sob o termo guarda-chuva de "ciência" ou "humanidade" (Reitsma, 2013).

Considerando a GIscience como uma área multidisciplinar, Blaschke e Merschdorf (2014) defendem que esta precisa acompanhar o desenvolvi- 
mento das Tecnologias da Informação e Comunicação (TIC), incluindo os diferentes sensores, a Internet das Coisas ou análise de Big Data para que os pesquisadores, ou GIscientists, possam fazer e responder perguntas relacionadas ao espaço e as representações espaço-temporais e assim tornarem-se distintos, por exemplo, dos cientistas da computação. Nesta perspectiva, vale ressaltar como fizeram autores como Blaschke, Stroble Donert (2011) e Blaschke et al. (2012) que a GIscience é um campo interdisciplinar relativamente novo de pesquisa e que novos campos para pesquisa em GIscience têm surgido.

Segundo Sinton (2016), conceitos de localização, representação e escala são centrais para as conexões entre pensamento espacial e GIscience. Em seu trabalho a autora explica que a maioria das pessoas dificilmente conhece a capacidade e as restrições tecnológicas de como um computador é capaz de representar as características do mundo natural e social, muito menos as diferenças entre os próprios modelos de dados espaciais. Em seus estudos, Smith e Mark (2010) observaram que as pessoas sentiam-se mais confortáveis identificando "características geográficas" como montanhas e rios enquanto elementos que podem ser retratados em um mapa, mas eram muito menos capazes de imaginar a representação cartográfica de um "objeto" ou "conceito" geográfico. Essa falta de familiaridade com a representação digital dos fenômenos geográficos, que existem para além do nível de observação direta independentemente da escala, afeta o modo como as pessoas entendem a natureza dos conjuntos de dados geográficos (Sinton, 2016). É neste contexto que as geotecnologias, em especial os sistemas de informação geográfica, podem ser empregadas como instrumentos potencializadores do desenvolvimento do pensamento espacial.

\section{SIG a serviço do desenvolvimento do pensamento espacial}

Um sistema de informação geográfica reflete muitas das funções e operações do pensamento espacial e pode servir como um meio de apoio para exemplificar os desafios e formas de incorporar o pensamento espacial na educação básica. Conceitualmente, um SIG consiste em um sistema integrado de hardware, software, usuários e procedimentos projetados para suportar a coleta, gerenciamento, manipulação, análise, modelagem e exibição de dados referenciados espacialmente sobre a superfície da Terra para resolução de problemas muitas vezes complexos, como os de planejamento e gestão territorial. Uma de suas funções é permitir a realização de operações em bancos de dados espaciais para resposta a perguntas genéricas relacionadas ao espaço geográfico e que podem motivar o desenvolvimento do raciocínio e pensamento espacial, tais como: O que está em...? Onde está...? O que mudou desde...? Que padrão espacial existe...? E se...? (NRC, 2006).
Embora sua utilização tenha sido disseminada, principalmente em função do surgimento e expansão do número de softwares livres de geoprocessamento e dos geoportais, vale lembrar que o uso adequado de um SIG, bem como de outros instrumentos como mapas, cartas e imagens de satélite, mesmo em formato analógico, depende da alfabetização geográfica do observador. No contexto do ensino, o sIG desempenha um papel importante que vai além da alfabetização geográfica. Ele permite compartilhar a alfabetização geográfica (conhecimento sobre geografia) com a alfabetização informacional (estratégias de busca de informações, avaliação crítica de fontes) levando dessa forma à alfabetização de informações geográficas (Figura 3), que para Zwartjes et al. (2016) consiste na posse de conceitos e habilidades mentais que permitem a um indivíduo entender e usar informações geográficas adequadamente e participar mais plenamente do debate público sobre questões relacionadas à Geografia.

Figura 3. Diagrama contextual para alfabetização em sistemas de informações geográficas.

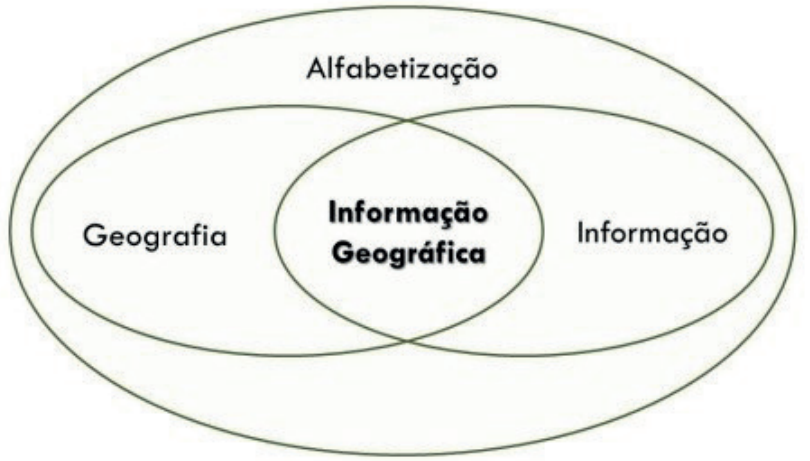

Fonte: Organizado pelos autores. Adaptado de Zwartjes et al. (2016)

A introdução do SIG no ensino pode ser defendida, principalmente, devido à sua influência na alfabetização digital, no desenvolvimento de habilidades e compreensão da realidade espacial, dentre outros, que por sua vez podem também influenciar na futura formação profissional dos estudantes. Johnson e Sullivan (2010) afirmam que o pensamento espacial desempenhará um papel significativo na economia baseada na informação do século 21 e acrescentam que as transformações tecnológicas e mudanças devido a novas ferramentas de mapeamento e novas fontes de dados, estão alterando objetivos educacionais e contextos de inovação, melhorando a análise de dados, a exploração de dados e a experiência do usuário.

Zwartjes et al (2016) consideram que o SIG e a GIScience apoiam o ensino e aprendizagem de Geografia e apresenta-se como um instrumento de grande eficiência para o estudo de problemas geográficos em diversas escalas, sendo essencial para os trabalhadores do conhecimento no século XXI. Além disso, no contexto educacional, o uso do SIG pode ser ainda valorizado devido à sua influência no processo da compreensão das teorias espaciais 
abstratas, no desenvolvimento do senso de localização e na motivação dos alunos em aprender devido à utilização de aplicativos e ferramentas que podem ser intuitivas e de fácil utilização. Conforme Florezano (2011),

Na medida em que o SIG possibilita integrar, analisar e espacializar (gerar mapas) informações locais, regionais e globais, ele torna-se um poderoso recurso didático. $O$ educador pode utilizar um SIG para inserir seu aluno no mundo tecnológico, tornar suas aulas mais dinâmicas e interessantes, bem como gerar seu próprio material didático para estudo do espaço local, de vivência do educando. O SIG dá oportunidade ao educador e educando de elaborar material que complemente os livros didáticos, de ligar o local com o global, apresentado nos livros didáticos (Florenzano, 2011, p.121).

No entanto, apesar de sua importância e influência, destaca-se aqui que o SIG não deve ser o único instrumento a ser explorado e que seu uso isolado ou descontextualizado também não terá um efeito positivo no processo de desenvolvimento do pensamento espacial. Assim, faz-se necessário destacar que,

(...) a aquisição da habilidade espacial não se constrói em poucas aulas, e jamais se desenvolverá plenamente se os programas continuarem a relegar o aprendizado das visualizações espaciais e cartográficas a momentos isolados do currículo escolar. Geralmente, os programas destinam momentos para o estudo dos mapas e da cartografia, atendendo apenas aos conteúdos de um currículo e não propriamente uma instrumentalização para o aprendizado dos diversos conteúdos da Geografia com o apoio dos mapas e representações cartográficas (Ferreira, 2013, p.77).

De acordo com Gryl et al. (2010) a aquisição tradicional de "habilidades de leitura de mapa" precisa ser revisitada e ajustada considerando que não apenas a construção mas também a interpretação de mapas exige um conjunto adicional de competências e que novas estratégias devem sem empregadas para utilização do instrumental cartográfico contemporâneo que contempla dentre outros, mapas online, globos virtuais e geoportais. Os autores defendem que habilidades como a orientação geral, direcional e topográfica bem como o entendimento da realidade a partir de diferentes escalas de representação são essenciais para uma futura participação ativa na sociedade. Os autores sugerem relacionar essa discussão ao campo mais amplo da educação para a cidadania espacial e recomendam três campos principais de competências que envolvem o domínio de competências técnicas e metodológicas para utilizar, avaliar, refletir e comunicar-se com e sobre o uso das representações espaciais de maneira adequada (Figura 4).

Figura 4. Competências para cidadania espacial

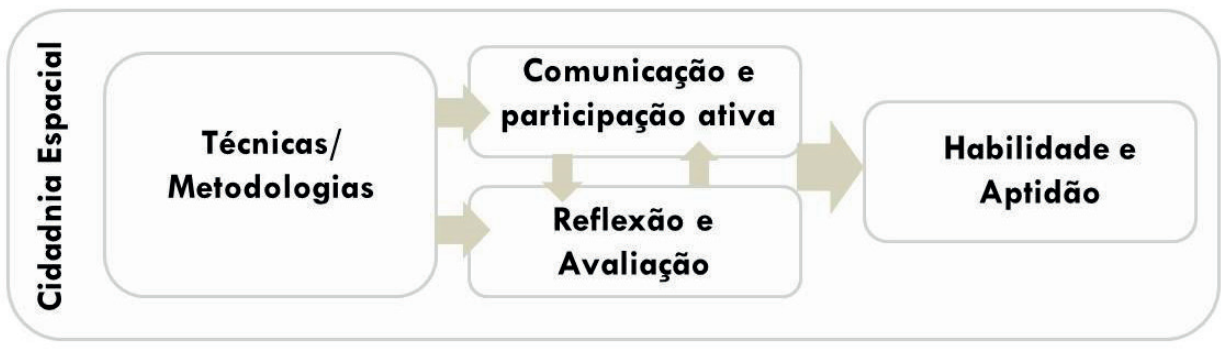

Fonte: Organização dos autores. Adaptado de Gryl et al. (2010).

Para esses três campos de competências, Gryl et al. (2010) identificam subconjuntos de competências que podem ser abordados mais diretamente em ambientes educacionais secundários, sendo que as competências técnicas não devem ser consideradas o objetivo final de uma educação para a cidadania espacial ativa, mas sim uma condição inicial para a formação de cidadãos espaciais ativos. Para comunicar e participar ativamente com informações espaciais, os autores recomendam que os alunos lidem com um conjunto de competências que inclui, dentre outras a construção de significados e a comunicação usando a informação geográfica.
Neste contexto, Goodchild e Janelle (2010) defendem que os estudantes devem ser educados segundo os padrões de um pensador espacial crítico, incluindo dentre outros:

- O potencial para contribuir com a compreensão espacial crítica para a pesquisa na interface entre disciplinas.

- A capacidade de trabalhar em equipe.

- A capacidade de explicar o contexto espaço-temporal de um estudo para não especialistas.

- A capacidade de transferir tecnologias espaciais e conceitos espaciais para pesquisa em diferentes domínios de conhecimento e conjuntos de problemas. 
Para os autores, atingir essas metas exigirá uma combinação de currículo convencional baseado em cursos, interação intensiva entre colegas, aprendizagem baseada em projetos e envolvimento com atividades em todo o espectro educacional (na comunidade e região, nacional e internacionalmente). Somam-se a essas recomendações a seleção adequada de ferramentas computacionais que podem ser utilizadas em sala de aula; pois, como defendido por autores como Silva (2013), tanto educador quanto educando precisam saber ler, entender e interpretar a informação espacial a eles apresentada e não apenas reproduzir um pressionar de botões de maneira mecanizada e ausente de uma abordagem conceitual adequada e reflexões críticas por parte daqueles que o fazem.

\section{Considerações}

Como apontado anteriormente, a discussão do papel das geotecnologias no desenvolvimento do pensamento espacial é uma tarefa complexa; pois não deve estar centrada apenas na utilização de um instrumental ou aparato tecnológico, mas sim envolver uma discussão mais profunda que considere a efetividade dessa relação.

As reflexões aqui apresentadas partem, em sua maioria, da necessidade do entendimento de que, mesmo sendo as geotecnologias consideradas como importantes instrumentos auxiliares das práticas didático-pedagógicas e potencializadores para o desenvolvimento do pensamento espacial, elas não devem ser usadas como um fim em si mesmo, pois dessa forma pouco contribui para a formação de um cidadão crítico e capaz de compreender e transformar a realidade em que vive; mas, devem ser utilizadas como um instrumental que possibilita a aplicação de conceitos, teorias e técnicas da representação cartográfica, do desenvolvimento cognitivo e de distintas áreas do conhecimento favorecendo a interdisciplinaridade e o desenvolvimento de novas formas de pensar e fazer Geografia. Apesar dos avanços tecnológicos e da disseminação crescente das geotecnologias no ambiente escolar como instrumentos auxiliares das práticas didático-pedagógicas ainda há alguns desafios a serem enfrentados para que estas possam ser aplicadas, de fato, no ensino de Geografia; dentre eles a precariedade da estrutura, equipamentos e adequação para uso das geotecnologias nas escolas e o despreparo de parte dos educadores para lidar com as tecnologias de informação e comunicação.

Cientes da extensão do debate que envolve a problemática aqui abordada, iniciamos uma discussão fundamentada nas reflexões que buscam apontar o estado da arte e os desdobramentos da aplicação das geotecnologias na rotina da sociedade contemporânea e no desenvolvimento do pensamento espacial crítico, e que são certamente passíveis de questionamentos e atualizações. Ainda há muito que se avançar.

\section{Referências}

Bednarz, R. e Lee, J. (2011). The components of spatial thinking: empirical evidence. Procedia Social and Behavioral Sciences, 21, 103-107.

Blaschke, T. e Merschdorf, H. (2014). Geographic information science as a multidisciplinary and multiparadigmatic field. Cartography and Geographic Information Science, 41(3), 196-213.

Blaschke, T., Strobl, J. e Donert, K. (2011). Geographic Information Science: Building a Doctoral Programme Integrating Interdisciplinary Concepts and Methods. Procedia - Social and Behavioral Sciences, 21, 139-146.

Blaschke, T., Strobl, J., Schrott, L., Marschallinger, R., Neubauer, F., Koch, A. e Beinat, E. (2012). Geographic Information Science as a Common Cause for Interdisciplinary Research. In J. Gensel, D. Josselin, e D. Wandenbroucke (Eds.), Bridging the Geographic Information Sciences (pp. 411-427). Springer.

Boroditsky, L. (2018). How language shapes the way we think. TED Talks. Disponível em:https://www.ted.com/talks/lera_boroditsky_ how_language_shapes_the_way_we_think/footnotes?referrer=playlist-the_most_popular_ted_talks_of_2018.

Castellar, S. (2017). Cartografia Escolar e o pensamento espacial fortalecendo o conhecimento geográfico. Revista Brasileira de Educação em Geografia, 7(13), 207-232.

Donert, K. (2008). Spatial Learning for Young European Citizen: a Geography toolkit. In S. Philippou e M. Beydioglu (Eds.), Exploring Europe and Ourselves: Geographies and Identities at Work. United Nations Development Programme.

Duarte, R. (2016). Educação Geográfica, Cartografia Escolar e Pensamento Espacial no segundo segmento do ensino fundamental (tese de doutorado). Universidade de São Paulo, São Paulo, Brasil.

Duarte, R. (2017). A linguagem cartográfica como suporte ao desenvolvimento do pensamento espacial dos alunos na educação básica. Revista Brasileira de Educação em Geografia, 7(13), 187-206.

Esquerdo, J., Cruz, S., Macário, C., Antunes, J., Silva, J. e Coutinho, A. (2014). Tecnologias da informação aplicadas aos dados geoespaciais. In S. Massruhá, M. Leite, A. Luchiari Junior, L. Romani (Eds.). Tecnologias da informação e comunicação e suas relações com a agricultura (pp. 139-156). Brasília: Embrapa.

Ferreira, R. (2013). A cartografia escolar e o desenvolvimento da habilidade espacial. Geografia Ensino \& Pesquisa, 17(1), 71-80. 
Florezano, T. (2011). Iniciação em Sensoriamento Remoto. São Paulo: Oficina de Textos.

Goodchild, M. (2004). The Validity and Usefulness of Laws in Geographic Information Science and Geography. Annals of the Association of American Geographers, 94(2), 300-303.

Goodchild, M. (2006). The Fourth R? Rethinking GIS Education. ArcNews. Disponível online.

Goodchild, M. (2009). Geographic information systems and science: today and tomorrow. Procedia Earth and Planetary Science, 1(1), 1037-1043.

Goodchild, M. (2011). Spatial thinking and the GIS User Interface. Procedia Social and Behavioral Sciences, 21, 3-9.

Goodchild, M. e Janelle, D. (2010). Toward critical spatial thinking in the social sciences and humanities. GeoJournal, 75(1), 3-13.

Gryl, I., Jekel, T. e Donert, K. (2010). Geoinformation and Spatial Citizenship. In T. Jekel, A. Koller, K. Donert e R. Vogler (Eds.), Learning with Geoinformation (pp. 2-11). Wichmann.

Johnson, A. e Sullivan, D. (2010). Geospatial Education at U.S. Community Colleges: Background, Challenges, and Opportunities. Urisa Journal, 22(2), 5-13.

Kerski, J. (2008). Developing Spatial Thinking Skills in Education and Society. ArcWatch. Disponível online.

Maciel, R. e Pinheiro, M. (2013). Pirâmides do conhecimento: abordagens comparativas entre ciência da informação e inteligência de estado. Pesquisa Brasileira em Ciência da Informação e Biblioteconomia, 8(1), 29-39.

Matias, L. (2004). Por uma economia política das geotecnologias. Scripta Nova, Revista Electrónica de Geografía y Ciencias Sociales, 8(170).

Metoyer S., Bednarz S. e Bednarz R. (2015). Spatial Thinking in Education: Concepts, Development, and Assessment. In O. Muñiz Solari, A. Demirci, J. Schee (Eds.), Geospatial Technologies and Geography Education in a Changing World. Advances in Geographical and Environmental Sciences (pp. 21-33). Tokyo: Springer.

National Research Council (NRC). (2006). Learning to think spatially: GIS as a support system in the $K$ - 12 curriculum. Washington: National Research Council Press.

Newcombe, N. (2010). Increasing Math and Science Learning by Improving Spatial Thinking. American Educator, 34(2), 29-35.
Piaget, J. (1983). A epistemologia genética. São Paulo: Abril Cultural.

Ramos, J. (2015). A ciência por trás da informação geográfica. MundoGeo. Disponível online.

Reitsma, F. (2013). Revisiting the 'Is GIScience a science?' debate (or quite possibly scientific gerrymandering). International Journal of Geographical Information Science, 27(2), 211-221.

Santos, M. (1978). Por uma Geografia Nova. São Paulo: Hucitec.

Silva, C. (2013). A cartografia em sala de aula na explicação do espaço geográfico (The cartography in the explanation of geographic space). ACTA Geográfica, 7(15), 55-68.

Sinton, D. (2016). Spatial Thinking and GIS. In H. Burte, T. Kauppinen, M. Hegarty (Eds.), Proceedings of the Workshop on Teaching Spatial Thinking from Interdisciplinary Perspectives (pp. 29-36). Santa Fe, NM.

Smith, B. e Mark, D. (2010). Geographical categories: an ontological investigation. Geographical Information Science, 15(7), 591-612.

Strobl, J. (2008), Digital Earth Brainware. In: Schiewe, J. e Michel, U. (eds.), Geoinformatics paves the Highway to Digital Earth (pp. 134-138). Universität Osnabrück, Osnabrück.

Tuan, Y. (1983). Espaço e lugar: a perspectiva da experiência. São Paulo: Difel.

Wakabayashi, Y. e Ishikawa, T. (2011). Spatial thinking in geographic information science: a review of past studies and prospects for the future. Procedia-Social and Behavioral Sciences, 21, 304-313.

Zwartjes, L., Lázaro, M., Donert, K., Sanchez, I., Gonzalez, R. e Wiśniewska, E. (2016). Literature review on spatial thinking. GI-Learner project. Erasmus+ Programme. Disponível online. 